\title{
INVESTIGATIONS ON HEAT TRANSFER CHARACTERISTICS OF POROUS TYPE COPPER HEAT SINK WITH BIFURCATIONS
}

\author{
T. Prabhu Thangavel${ }^{1}$, C. Ajay Sekar ${ }^{2}$
}

\begin{abstract}
The lotus type porous copper heat sink has a higher heat transfer capacity as compared to micro channel heat sink for same size. Many studies on this heat sink show that its heat transfer capacity can be further improved by improving design of heat sink. This study investigates the heat transfer and flow characteristics of porous heat sink with different designs of internal bifurcations under both laminar \& turbulent flow and studies the effect of such bifurcations on heat transfer of heat sink. The results of heat sink with bifurcations are compared with that of heat sink without bifurcations which show that for case of both laminar \& turbulent flow, the heat sink with bifurcation showed better thermal performance as compared to heat sink without bifurcations.
\end{abstract}

Keywords: Porous Type Copper Heat Sink, Laminar Flow, Turbulent Flow, Bifurcations, Characteristic Curves.

\section{INTRODUCTION.}

For quite a long time, there has been focus on reducing the size and volume of electronic devices. On other hand with development of technology and need for more complex functions, there has been development on integrating different functions of electronic devices into single component. This has resulted in increasing the amount of heat released by such devices. Such situations call for design of cooling systems with good thermal performance but should be lesser in weight. This cannot be achieved by traditional methods of cooling such as use of fans with air as coolant as it occupies more space and there occurs more noise with increasing size. Thus, this emphasizes on need of such studies on increasing thermal performance of cooling systems such has heat sinks, heat exchangers etc.

\section{Literature Review}

Gongnan Xie et al [1] improved the thermal heat transfer characteristics of microchannel heat sinks by use of internal Y shaped bifurcations. Gongnan Xie et al also studied on effect of angle of arms of bifurcations and $\mathrm{Y}$ shaped bifurcation length on overall performance of heat sink. The results of study showed that the microchannel heat sink with bifurcations have a better thermal performance when compared with microchannel heat sink without bifurcations. With increase in velocity, the thermal performance of microchannel heat sink with bifurcations was found to decline sharply for corresponding velocities in microchannel heat sink without bifurcations. Thus, the study shows internal bifurcations is a possible method to increase them performance of a heat sink.

The work by Fengli Zhang et al [2] considered different constructural designs of bifurcations for microchannel heat sink and investigated the heat transfer results for different inlet velocities ranging from $0.6 \mathrm{~m} / \mathrm{s}$ to $1.6 \mathrm{~m} / \mathrm{s}$. The numerical results obtained from study show that heat sinks with multiple bifurcations have a better thermal performance compared with straight channel microchannel heat sink. The study by Zhang et al [2] showed that use of multiple bifurcations can help reduce thermal resistance of heat sinks. The results obtained form study thus show that proper design of bifurcation can help improve thermal performance.

A study on microchannel heat sink with multiple bifurcations under laminar flow was done by Shian Lie et al [3]. The bifurcation length of five different cases was considered and its thermal characteristics was studies.

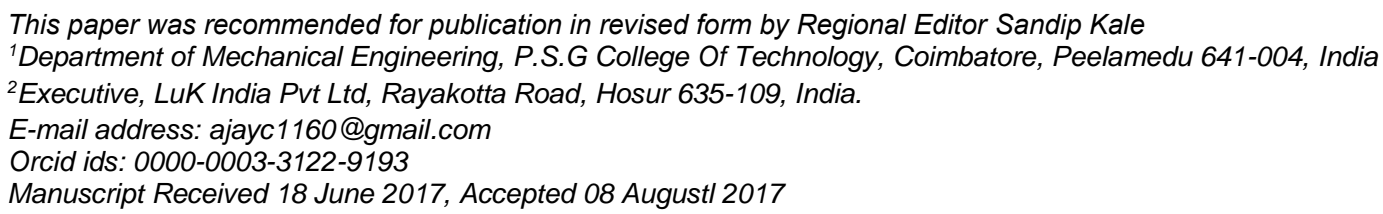


The study obtained local pressure profiles, velocity and temperature profiles are presented which show that heat sinks with long length bifurcations is better as compared to microchannel heat sinks without bifurcations.

D.R.S. Raghuraman et al [4] studied the effect of aspect ratio on thermal performance of rectangular shaped microchannel heat sink using CFD code. The optimum ratio at which heat transfer is maximum is obtained numerically. The thermal performance of heat sink with an aspect ratio of 20 was found to have superior as compared to other cases. At the same time the microchannel heat sink with aspect ratio of 20 was found to have a higher pressure drop as compared with other cases. The heat sink with aspect ratio of 30 was found to be optimum as it had higher heat removal date.

Deborah V. Pence [5] conducted a study on reducing the pumping power and temperature of wall by use of fractal like branching networks.Fractal like heat sinks showed a 60\% lower pressure drop and 30 degree Celsius lower temperature when compared to that of ordinary .

The study conducted by S.M. Senn et al [6] shows the importance of bifurcations and tree like nets on thermal performance of heat sinks numerically. The study shows that secondary flows are initiated at bifurcations which help to increase heat transfer characteristics by laminar mixing as compared to conventional serpentine structure without bifurcations.

Heng Ren [7] studied about effect of different types of structures in microchannel on cooling capacity of heat sink. Three types of microchannel designs were considered and temperature distribution and pressure drop on microchannel heat sink with different structures was investigated and it was found that heat sink with reverting structure was found to have considerable effect on temperature and pressure drop of heat sink. The use of reverting microstructure in heat sink was found to reduce maximum temperature and increase pressure drop in heat sink.

Xueye Chen et al [8] performed a numerical analysis of various types of micromixers based on fractal tree like network. The study showed that bifurcation angle is an important parameter that affects performance of heatsink with bifurcation. The numerical analysis results showed that bifurcation angle of 90 degrees was found to be optimum.

Yongping Chen et al [9] focused on studying effect of fractal branching channel network for heat sinks which showed that the effect of fractal branching network on pressure drop is negligible and it can increase the total heat transfer capacity of heat sink. Also a larger number of fractal network or larger length of fractal network can help increase magnitude of heat transfer appropriately.

Bladimir Ramos-Alvarado et al[10] performed numerical study on heat sinks with conventional and new flow channel configurations with flow regime as laminar. The study concludes that use of bifurcations on heat sinks helps to reduce temperature difference on heat sink per unit heat flux and a low thermal resistance.

The performance of an heat exchanger with tree like flow passages was examined by David Calamas and John Baker [11].They found that the flow network caused by hierarchical bifurcating flow passages resulted in a pressure drop that is nearly two orders in magnitude smaller than traditional compact heat exchanger designs but resulted an increase in bulk temperature of fluid larger in magnitude as compared with microscale flow network that was studied. They also found that bifurcation angle had an important influence on pressure drop and wall temperature distribution because of flow separation and recirculation zones that are caused by such bifurcations.

A study was made on design of microchannel heat sink based on natural designs. Carlos Alberto RubioJimenez et al [12] found that temperature contours obtained on heat sinks studied were non-homogeneous in nature. The design of heat sink corresponding to case 2-A was found to be most suitable design since the temperature distribution obtained on this design was found to be below the design point. A significant finding from this study is that the velocity of fluid can be increased by decreasing hydraulic diameter.

An experimental study was done by R.S.Mali et al [13] on heat sink with leaf like structure and found that leaf like structure resulted an increase in heat transfer capacity of heat sink. An important observation from the study is that the heat transfer capacity of heat sink with leaf like design is increased significantly for lower mass flow rates when compared with conventional type microchannel heat sink under the same conditions.

W. Escher et al [14] determined performance of bifurcating heat sink with that of parallel microchannel heat sink for same conditions and found that parallel microchannel heat sinks performance superior to that of bifurcating heat sink. The heat transfer capacity of parallel microchannel heat sink was found to be four times higher than bifurcating heat sinks along with a higher heat transfer coefficient because of dense packed nature of parallel microchannel heat sink thus allowing the heat sink to transfer heat more efficiently. 


\section{DESCRIPTION OF LOTUS TYPE POROUS COPPER HEAT SINK}

The lotus type porous copper heat sink is a kind of heat sink similar to the microchannel heat sink with the exception that porous heat sink has long cylindrical pores as compared with long channels of a microchannel heat sink. The heat is absorbed by working fluids such as air flowing through pores. The heat sink is shown in figure 1. In reality the pores of copper heat sink are not exactly cylindrical in nature. Tetsuro Ogushilet al [15] showed that lotus type porous heat sink has a heat transfer capacity nearly four times as compared with conventional groove fins. It must be noted that to reduce computational time, only small size of heat sink has been considered in this study. Since heat transfer is a property dependent on area, with larger surface area, a better temperature gradient can be obtained.
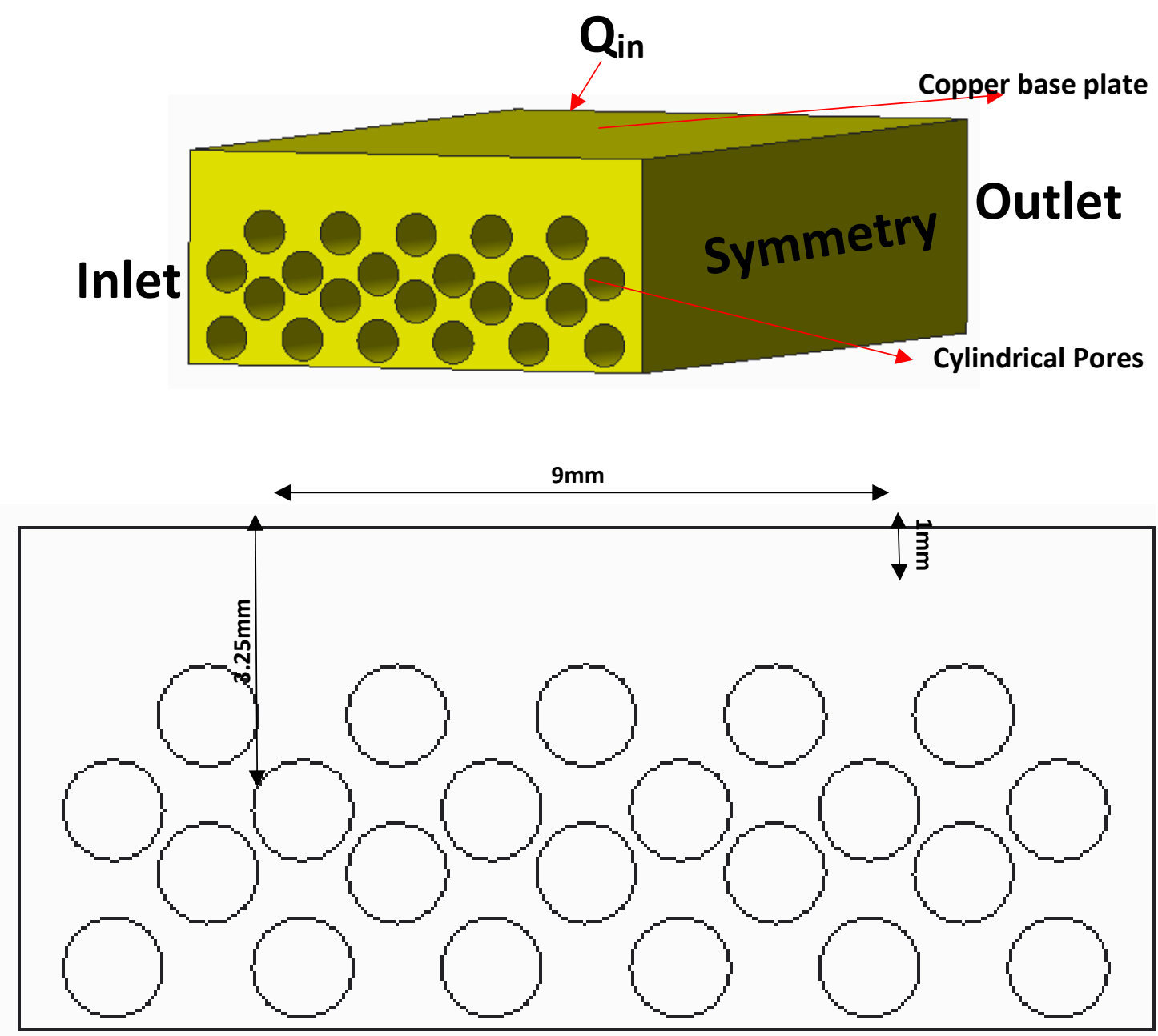

Figure 1. Lotus type porous heat sink used in this study along with dimensions and boundary conditions

\section{Experimental Study}

The specimen required for testing is prepared first by cutting required size of heat sink cut from ingot and then polishing it using Sic paper. After this process, the base plate made of copper is attached to lotus type heat sink using welding technique. Figure 2 below shows schematic diagram of experimental apparatus used in testing of lotus type porous heat sink. The experimental apparatus consists of two pipes which supply required fluid to cool the heat sink through use of pump. The heat sink is heated with the help of a heating unit. The temperature at base plate of heat sink was measured using $\mathrm{T}$ type thermocouple. The two junctions of thermocouple were stuck to surface of heat sink using adhesive. The heating unit consisted of a knob which could be adjusted to provide required level of heat flux to heat sink. 


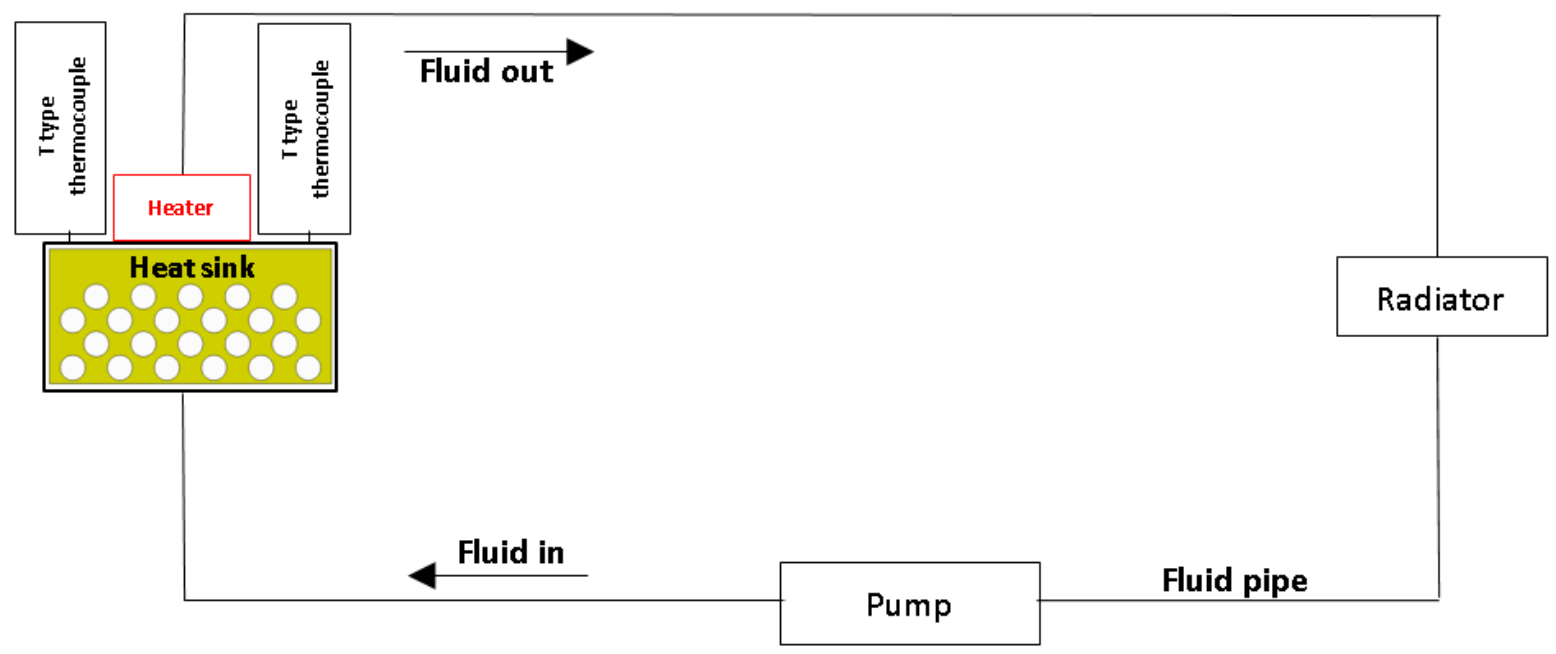

Figure 2. Schematic overview of experimental setup

The values of temperature obtained using experimental setup for both laminar and turbulent flow is tabulated in table 1 below. The error percentage between experimental and analytical results was found to be around $10 \%$. This difference can be attributed to the fact that in real lotus type porous copper heat sink the pores are not completely spread throughout length of heat sink thus resulting in higher temperature values.

Table 1. Experimental value and numerical values of temperature for laminar and turbulent flows

\begin{tabular}{|c|c|c|c|c|c|c|c|c|} 
& \multicolumn{4}{|c|}{ Type: Laminar flow } \\
\hline \multirow{3}{*}{ Reynolds number } & \multicolumn{4}{|c|}{$\begin{array}{c}\text { Temperature (K) } \\
\text { Numerical results }\end{array}$} & \multicolumn{5}{c|}{$\begin{array}{c}\text { Temperature (K) } \\
\text { Experimental results }\end{array}$} \\
\cline { 2 - 10 } & Case a & Case b & Case c & Case d & Case a & Case b & Case c & Case d \\
\hline 100 & 363 & 362 & 368 & 350 & 400 & 409 & 406 & 395 \\
\hline 300 & 320 & 320 & 320 & 323 & 347 & 340 & 335 & 361 \\
\hline 500 & 312 & 313 & 313 & 316 & 342 & 351 & 352 & 355 \\
\hline 700 & 323 & 311 & 309 & 313 & 363 & 326 & 343 & 324 \\
\hline 900 & 310 & 310 & 305 & 311 & 328 & 329 & 319 & 330 \\
\hline
\end{tabular}

\begin{tabular}{|c|c|c|c|c|c|c|c|c|} 
& \multicolumn{4}{|c|}{ Type: Turbulent flow } \\
\hline \multirow{3}{*}{ Reynolds number } & \multicolumn{4}{|c|}{$\begin{array}{c}\text { Temperature (K) } \\
\text { Numerical results }\end{array}$} & \multicolumn{5}{c|}{$\begin{array}{c}\text { Temperature (K) } \\
\text { Experimental results }\end{array}$} \\
\cline { 2 - 11 } & Case a & Case b & Case c & Case d & Case a & Case b & Case c & Case d \\
\hline 4100 & 329 & 317 & 321 & 398 & 360 & 342 & 366 & 425 \\
\hline 4300 & 321 & 314 & 315 & 318 & 352 & 354 & 354 & 349 \\
\hline 4500 & 314 & 313 & 310 & 312 & 335 & 350 & 337 & 335 \\
\hline 4700 & 307 & 306 & 305 & 305 & 330 & 342 & 331 & 333 \\
\hline 4900 & 303 & 302 & 302 & 302 & 318 & 321 & 326 & 322 \\
\hline
\end{tabular}

\section{NUMERICAL ANALYSIS}

\section{Model Description and Mesh Validation}

The geometric model used in this study is shown in figure 2 above. In order to reduce the computational time and complexity of the problem, only a small portion of porous type copper heat sink is considered. The following are assumptions made in this study

(1) The process is assumed to be a steady state process.

(2) The physical properties of material remain constant and do not change with variables such as temperature. 
(3) Heat transfer due to radiation is neglected.

(4) The effect of surface tension in liquid is neglected.

(5) The material is homogeneous in nature and does not have any impurities.

The slip in velocity between fluid and solid is assumed to be zero. Uniform velocity and pressure distribution is assumed to be present at entrance of fluid into heat sink. The fluid is assumed to leave the heat sink with atmospheric pressure. A constant heat flux is provided at top wall of heat sink. It is assumed that side walls of heat sink have zero temperature and velocity gradients.

The side view of different designs of heat sink with bifurcations used in this study is shown in figure 3 below.

After importing geometry and meshing it, finite volume method and fluent code are used to solve corresponding governing equations with boundary conditions to get temperature and pressure fields. Convergence criteria is chosen in such a way that residual value is less than $10^{-6}$. In order to avoid influence of mesh on results obtained a grid independence study was performed and results obtained are tabulated below. The study has been carried out with 3 million number of elements since the error percentage was found to be very less. A similar number of elements are used for both laminar and turbulent flow.

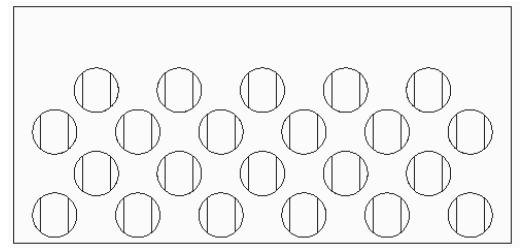

(a)

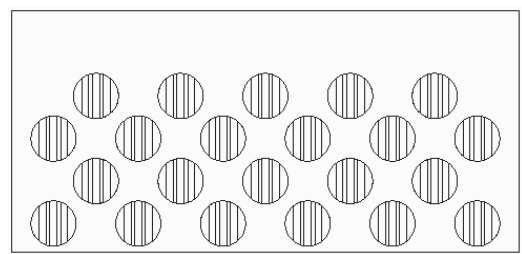

(b)

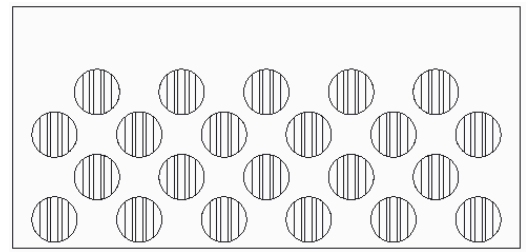

(c)

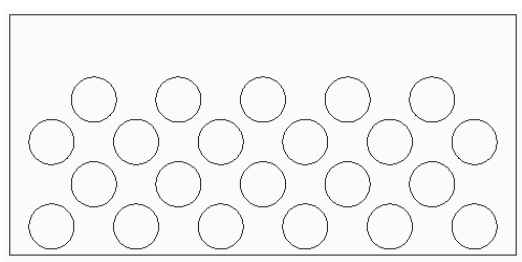

(d)

Figure 3. (a). Heat sink with single bifurcation, (b). Heat sink with multiple bifurcations with extreme bifurcations having depth of $0.0045 \mathrm{~m}$, (c). Heat sink with multiple bifurcations with centre bifurcation having depth of $0.0045 \mathrm{~m},(\mathrm{~d})$. Heat sink with no bifurcations

Table 2. Mesh independence test results

\begin{tabular}{|c|c|c|}
\hline Number of elements & Temperature & Error \% \\
\hline 1.25 million & $410 \mathrm{~K}$ & - \\
\hline 2.5 million & $404 \mathrm{~K}$ & $1.2 \%$ \\
\hline 3 million & $400 \mathrm{~K}$ & $0.9 \%$ \\
\hline
\end{tabular}

\section{RESULTS AND DISCUSSIONS}

\section{Pressure Drop Characteristic -Laminar Flow}

Fig .4 below shows the pressure drop characteristics of different designs of heat sink with bifurcations. As seen for the case of laminar flow, the heat sink with no bifurcations corresponding to case $d$ has a lower pressure drop as compared to all other heat sinks with bifurcations. This is because the bifurcation causes the fluid to separate resulting in separation zones thus resulting in higher pressure drops. From the graph, it is also seen that with increase in Reynolds number there seems to be an increase in pressure drop as well and the velocity at which the pressure drop reaches maximum is based on design of bifurcation in heat sink and on physical properties of fluid. 


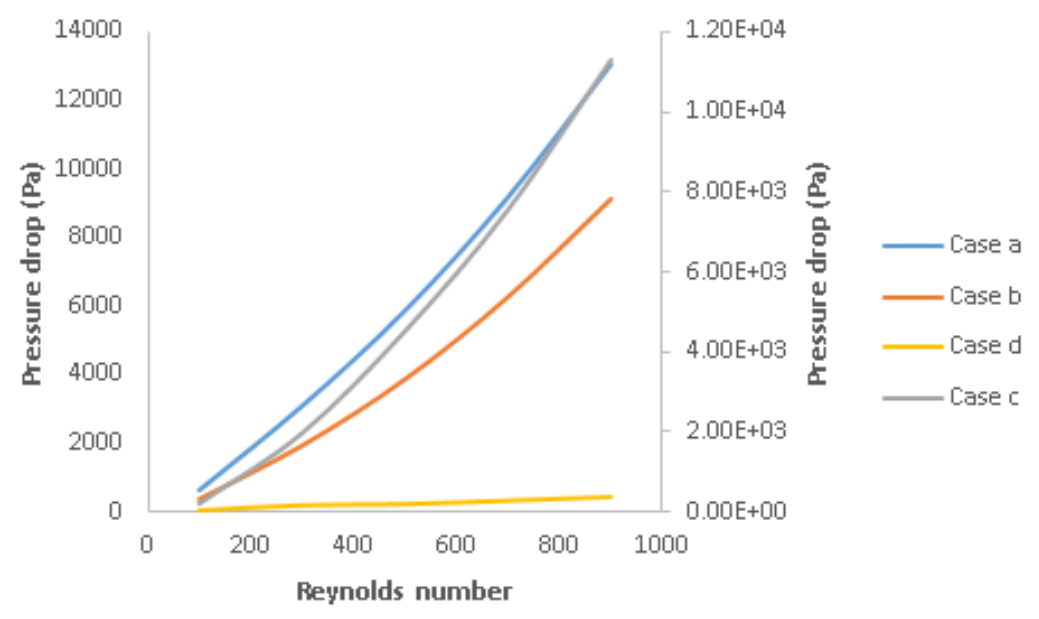

Figure 4. Pressure drop Vs Reynolds number graph for laminar flow

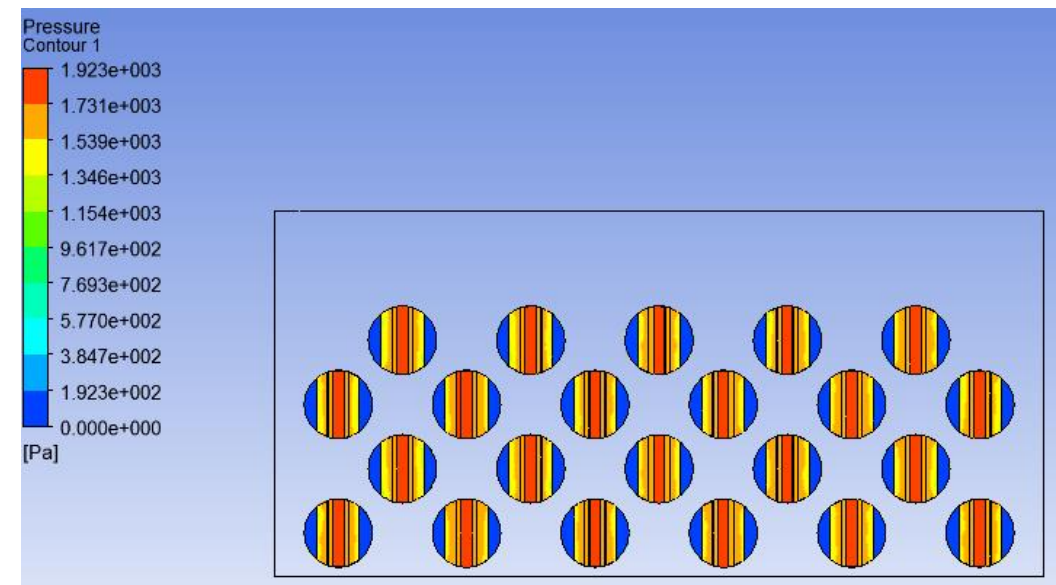

Figure 5. Pressure contours for Case $d$ with velocity $v-6 \mathrm{~m} / \mathrm{s}$

\section{Thermal Characteristics- Laminar Flow}

The thermal contours obtained for different cases is shown in fig. 5 below. The temperature seems to be maximum at velocity of $2 \mathrm{~m} / \mathrm{s}$ for heat sink without bifurcation. For call cases, it has observed that with increase in velocity of fluid, there occurs a steady drop in temperature. However, the temperature seems to reach a minimum value $305 \mathrm{~K}$ for case $\mathrm{c}$ and $310 \mathrm{~K}$ for case $\mathrm{b}$ at higher velocities. The heat sinks with bifurcations have a larger temperature gradient because the bifurcations result in boundary layer separations of fluid near its walls which causes an increase in heat transfer rate thus resulting in a temperature drop.

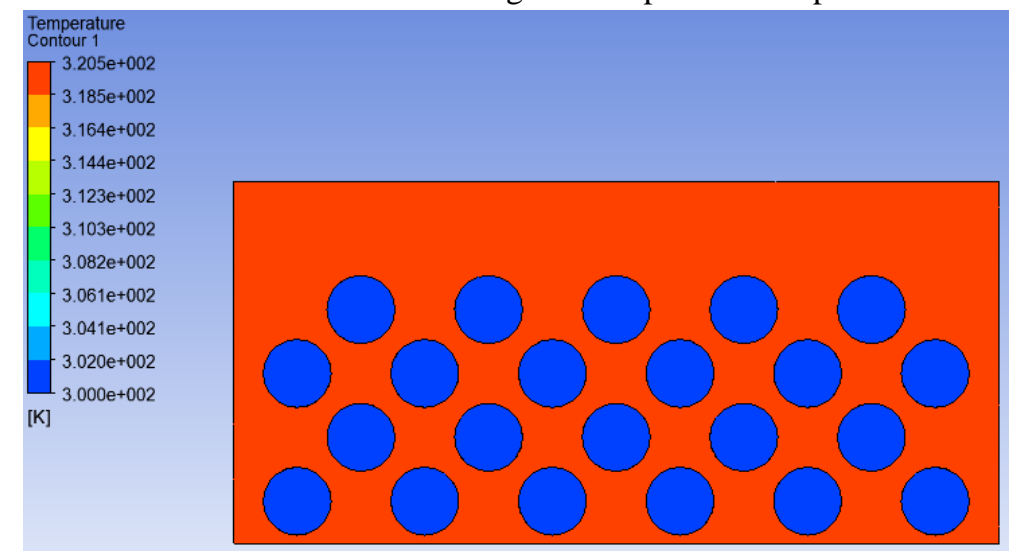

Figure 6. Temperature contours for Case $b$ with velocity $v-6 m / s$ 


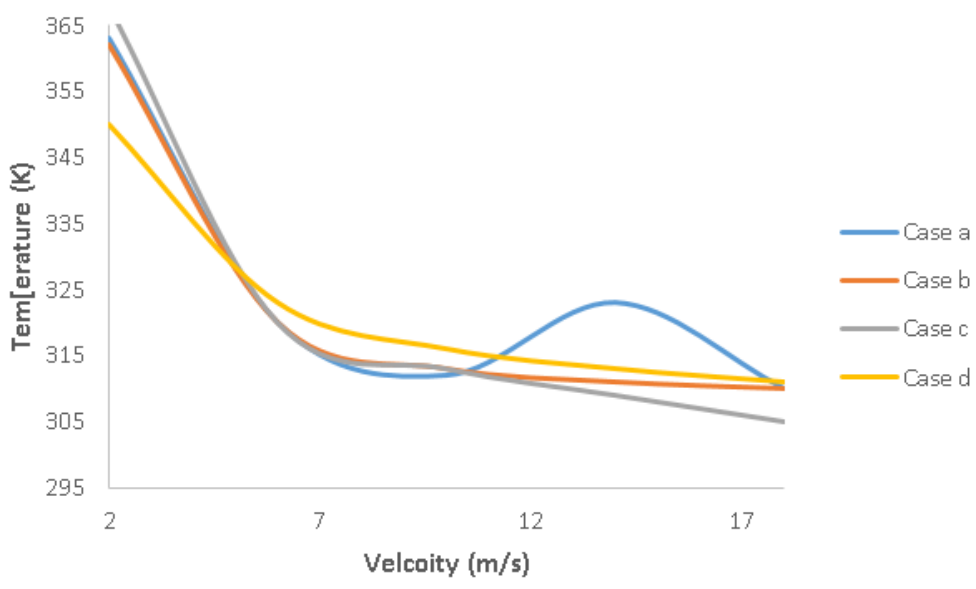

Figure 7. Temperature Vs velocity graph for laminar flow

\section{Pumping Power - Laminar Flow}

It can be observed from the graph that pressure drop has a strong correlation with pumping power. The pumping power increases linearly with increase in Reynolds number. The pumping power is found to be minimum for case $d$ as expected because this heat sink does not have any bifurcations. The other heat sinks with bifurcations restrict the easy flow of fluid which causes an increase in pressure. The pumping power is found to increase more steeply for case a which has a single bifurcation and pumping power was also found to be maximum for this case since larger surface area of bifurcation causes a higher pressure drop. The pressure drop for other cases was found to be intermediate between two cases.

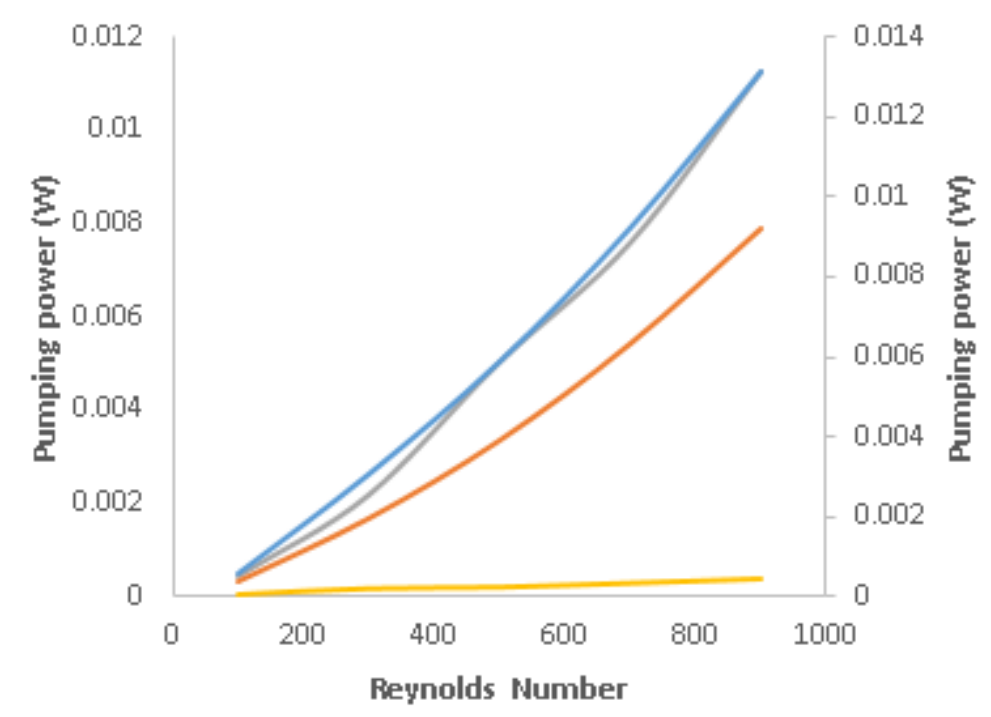

Figure 8. Pumping power Vs Reynolds number graph for laminar flow

\section{Pressure Drop Characteristic -Turbulent Flow}

Fig. 9 shows pressure drop graph for case of turbulent flow for all four cases. In graph the pressure drop increases with Reynolds number parabolically and reaches a maximum point. On further increasing the Reynolds number, the pressure drop begins to dip and reaches a minimum point. This nature of curve continues with further increase in velocity. Case $d$ has a minimum pressure like laminar case as it does not have any bifurcations. An important observation to be made between pressure drop characteristics of laminar and turbulent flow is that for laminar flow, there is no dip in pressure drop with increase in velocity for laminar flow which is not the case for turbulent flow. 

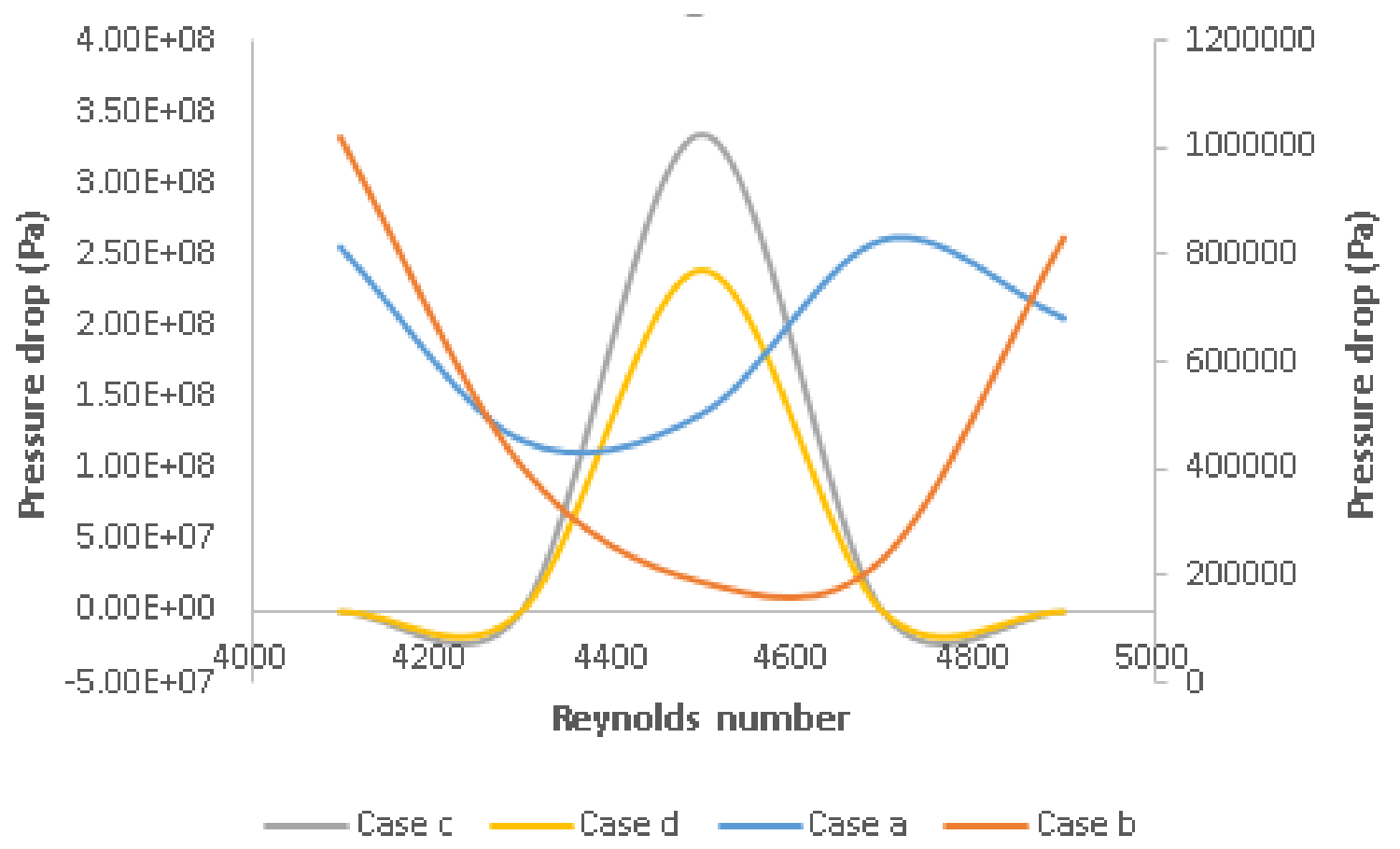

Figure 9. Pressure drop Vs Reynolds number graph for turbulent flow

\section{Thermal Characteristic -Turbulent Flow}

The thermal characteristics for turbulent flow were found to be similar to that of laminar flow with certain differences. For velocity of $82 \mathrm{~m} / \mathrm{s}$, the heat sinks with bifurcations corresponding to cases $\mathrm{a}, \mathrm{b}$ and $\mathrm{c}$ have a lower temperature while heat sink without any bifurcation has a higher temperature. The important difference between thermal characteristics of laminar and turbulent flow is that the temperature gradient in case of turbulent flow is more initially when compared to that of laminar flow. Also, it is seen that with continuous increase in velocity, all heat sinks approach towards a similar kind of temperature as the heat sink attains steady state. Although all heat sinks approach towards a similar kind of temperature with higher velocities, it is possible to take advantage of the fact that the heat sink with bifurcations that have a lower temperature at a lower velocity as compared to heat sink without any bifurcations. Case $\mathrm{c}$ was found to have a minimum temperature of $302 \mathrm{~K}$ at higher velocity.

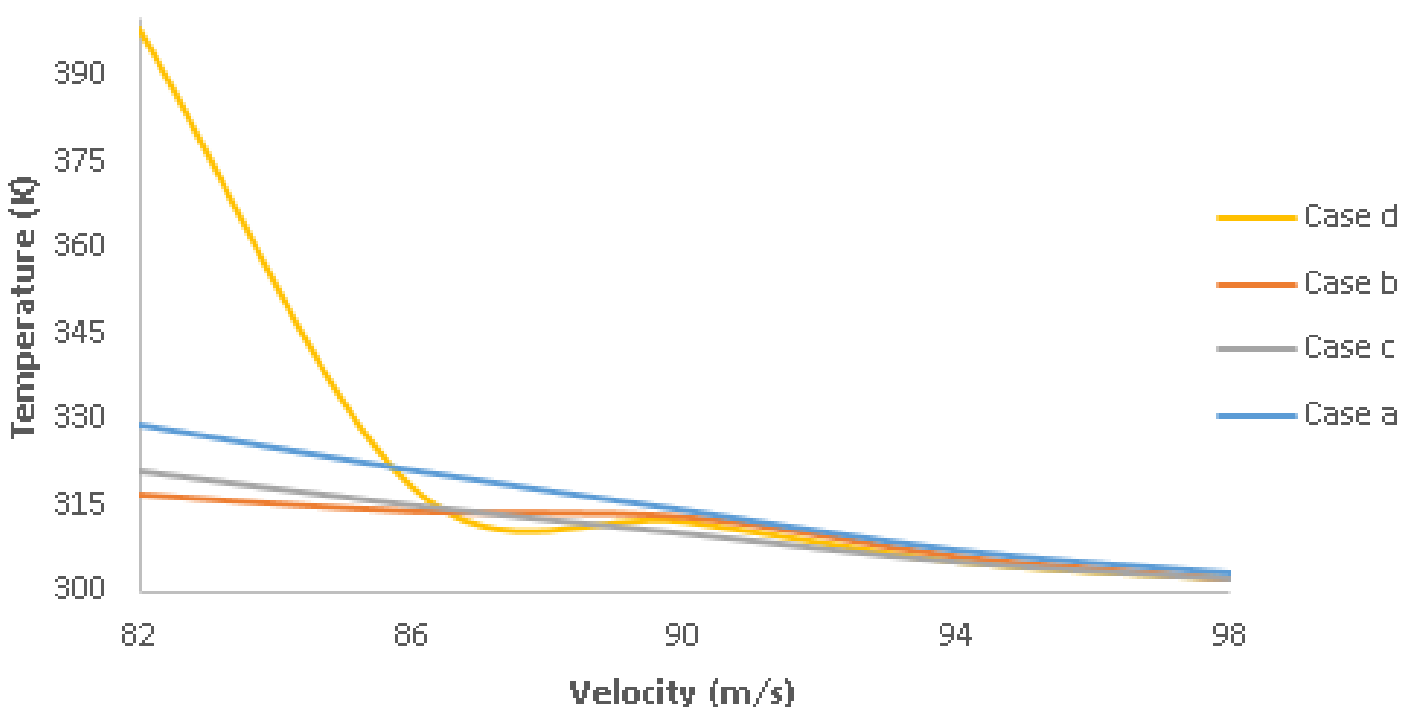

Figure 10. Temperature Vs Velocity graph for turbulent flow 


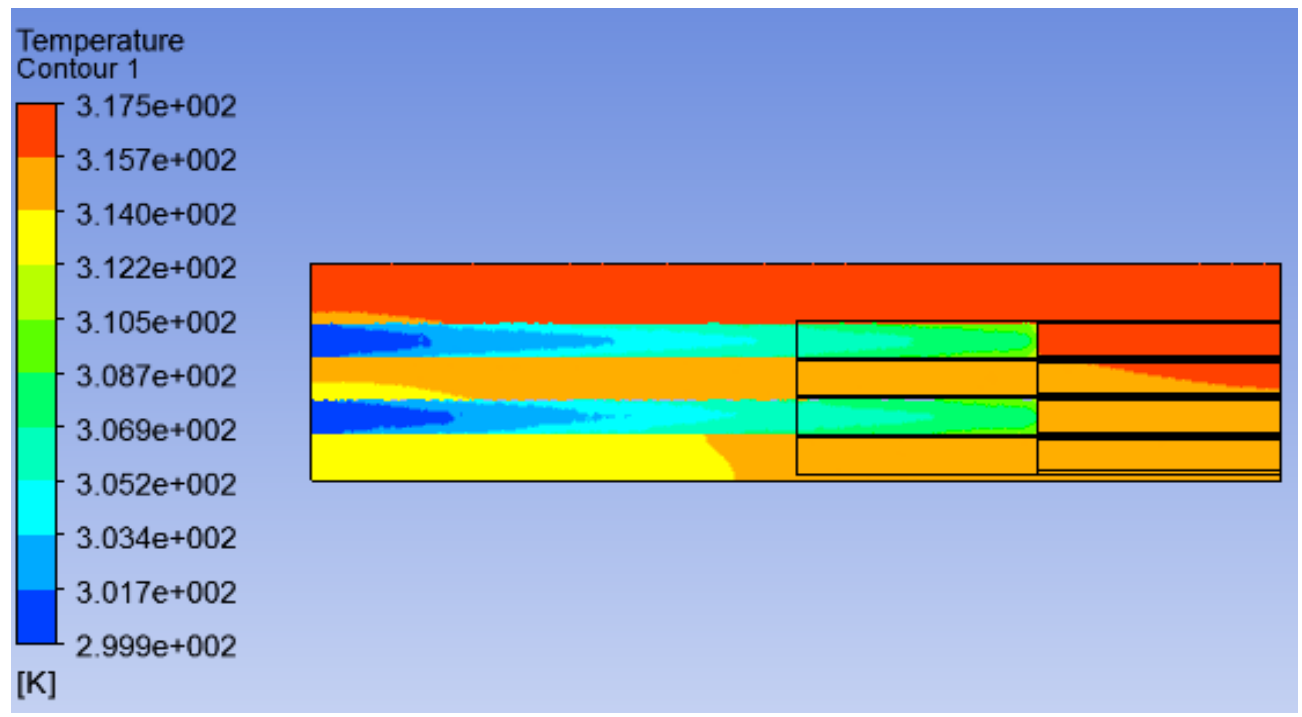

Figure 11. Temperature contours for case $b$ with velocity $82 \mathrm{~m} / \mathrm{s}$

\section{Pumping Power - Turbulent Flow}

As seen in fig. 11, for the case of turbulent flow pumping characteristics, like laminar flow the pressure drop of case $d$ is less and thus it has a lower pumping power. Like pressure characteristics of laminar flow, there seems to be a maximum and minimum points at which pumping power reaches maximum and minimum values accordingly.

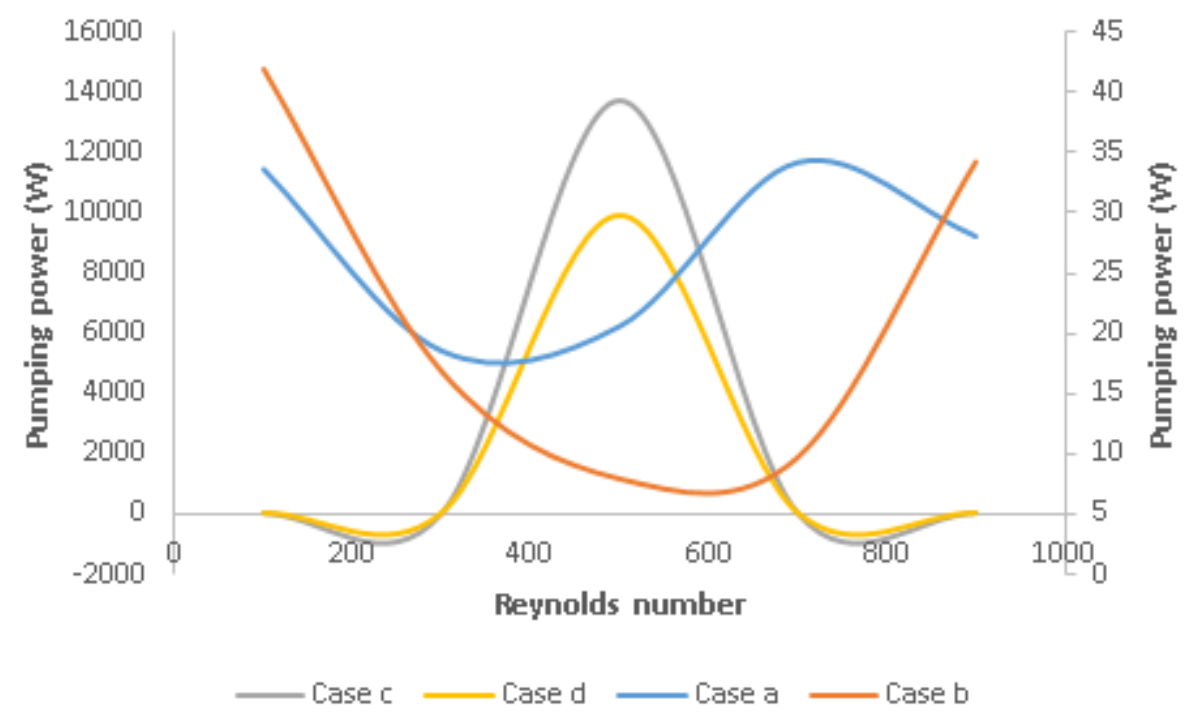

Figure 11. Pumping power Vs Reynolds number for turbulent flow

\section{SUMMARY AND CONCLUSIONS}

In this paper, bifurcations are designed for porous type copper heat sink. The effect of bifurcations on performance of porous type heat sink has been examined in this study for both laminar and turbulent flow. The following discussions could be concluded:

(1) The calculated results show that heat sink with bifurcations show a better thermal performance when compared with other heat sink without bifurcation. Thus, bifurcations provide a possible potential to further increase the heat transfer capacity of porous heat sink. A proper design of bifurcation can result in optimum pressure drop and pumping power thus resulting in optimum thermal performance. 
(2) For both laminar and turbulent flows, the temperature drop increase with respect to increase in Reynolds number. However, for case of turbulent flow, the temperature drop is more as compared with that of laminar flow.

(3) The pumping power for case of laminar flow increases steeply for heat sinks with bifurcations while for case of turbulent flow, the pumping power attains a maximum value at certain velocity and reaches a minimum value at certain velocity which in turn is decided by design of bifurcation and properties of cooling fluid. A similar kind of observation was found for pressure drop as well for both cases.

\section{CONFLICT OF INTEREST}

None

\section{REFERENCES}

[1] Xie G ,Shen H, Wang CC. Parametric study on thermal performance of microchannel heat sinks with internal vertical Y-shaped bifurcations. International Journal of Heat and Mass Transfer, 2015; 90: 948958. Doi: https://doi.org/10.1016/j.ijheatmasstransfer.2015.07.034

[2] Xie G, Zhang F, Sundén B, Zhang W. Constructal design and thermal analysis of microchannel heat sinks with multistage bifurcations in single-phase liquid flow. Applied Thermal Engineering, 2014; 62(2) 791-802. Doi: https://doi.org/10.1016/j.applthermaleng.2013.10.042

[3] Xie G, Li S, Sunden B, Zhang W, Li H. A Numerical Study of the Thermal Performance of Microchannel Heat Sinks with Multiple Length Bifurcation in Laminar Liquid Flow. Numerical Heat Transfer, Part A: Applications: An International Journal of Computation and Methodology, 2013; 65(2): 107-126. Doi: https://doi.org/10.1080/10407782.2013.826084

[4] Raghuraman DRS, Raj RTK, Nagarajan PK, Rao BVA. Influence of aspect ratio on the thermal performance of rectangular shaped micro channel heat sink using CFD code. Alexandria Engineering Journal, 2016; 56(1): 43-54. Doi: https://doi.org/10.1016/j.aej.2016.08.033

[5] Pence D. Reduced Pumping Power And Wall Temperature In Microchannel Heat Sinks With FractalLike Branching Channel Networks. Microscale Thermophysical Engineering, 2003; 6(4): 319-330. Doi: https://doi.org/10.1080/10893950290098359

[6] Senn SM, Poulikakos D. Laminar mixing, heat transfer and pressure drop in tree-like microchannel nets and their application for thermal management in polymer electrolyte fuel cells. Journal of Power Sources, 2004; 130(1): 178-191. Doi: https://doi.org/10.1016/j.jpowsour.2003.12.025

[7] Ren H. Numerical study of the heat transfer performance of heat sinks with different microchannel structures. International Journal of Science, Engineering and Technology Research, 2016; 5(7): 24262428.

[8] Chen X, Zhang Z, Yi D, Hu Z. Numerical studies on different two- dimensional micromixers basing on a fractal- like tree network. Microsyst Technol, 2017; 23: 755-763. Doi: https://doi.org/10.1007/s00542015-2742-X

[9] Chen Y, Cheng P. Heat transfer and pressure drop in fractal tree-like microchannel nets, International Journal of Heat and Mass Transfer. 2002; 45(13) 2643-2648. Doi: https://doi.org/10.1016/S00179310(02)00013-3

[10] Ramos-Alvarado B, Li P, Liu H, Hernandez-Guerrero A. CFD study of liquid-cooled heat sinks with microchannel flow field configurations for electronics, fuel cells, and concentrated solar cells. Applied Thermal Engineering, 2011; 31(14): 2494-2507. Doi: https://doi.org/10.1016/j.applthermaleng.2011.04.015

[11] Calamas D, Baker J. Performance of a Biologically Inspired Heat Exchanger with Hierarchical Bifurcating Flow Passages. Journal Of Thermophysics And Heat Transfer, 2013; 27(1): 80-90. Doi:https://doi.org/10.2514/1.T3950

[12] Rubio-Jimenez CA, Hernandez-Guerrero A, Rubio-Arana JC, Kandlikar S. Natural Patterns Applied to the Design of Microchannel Heat Sinks. Proceedings of the ASME 2009 International Mechanical Engineering Congress \& Exposition, IMECE2009, November 13-19, Lake Buena Vista, Florida, USA. Doi: https://doi.org/10.1115/IMECE2009-12047 
[13] Mali RS, Acharya AR, Gaikwad P. Experimental Study Of Heat Transfer In Micro-Channel Heat- Sink With Leaf- Like Pattern. International Journal of Advance Research In Science And Engineering, IJARSE, 2015; 4(4): 211-216.

[14] Escher W, Michel B, Poulikakos D. Efficiency of optimized bifurcating tree-like and parallel microchannel networks in the cooling of electronics. International Journal of Heat and Mass Transfer 2009; 52 (5): 1421-1430. Doi: https://doi.org/10.1016/j.ijheatmasstransfer.2008.07.048

[15] Lin L, Deng MX, Zhang XX, Wang XD. Numerical analysis and parametric study of multilayered microchannel heat sinks. Advances in Mechanical Engineering 2015; 7(7): 1-10 Doi:https://doi.org/10.1177\%2F1687814015594124

[16] Ogushi T, Chiba H, Nakajima H. Development of Lotus-Type Porous Copper Heat Sink. Special Issue on Porous and Foamed Metals - Fabrication, Characterization, Properties and Applications Materials Transactions, 2006; 47(9): 2240-2247.

[17] Delavar MA, Azimi M. Using Porous Material for Heat Transfer Enhancement in Heat Exchangers: Review. Journal of Engineering Science and Technology Review, 2013; 6(1): 14-16.

[18] Alharbi AY, Pence DV, Cullion RN. Fluid flow through microscale fractal-like branching channel networks. Journal of Fluids Engineering, 2003; 125(6) :1051-1057. Doi: https://doi.org/10.1115/1.1625684

[19] Mou N. Numerical investigations on fluid flow and heat transfer characteristic in oblique fin microchannel array. International Journal of Heat and Mass Transfer, 2014;

[20] Lee YJ, Singh PK, Lee PS. Fluid flow and heat transfer investigations on enhanced microchannel heat sink using oblique fins with parametric study. International Journal of Heat and Mass Transfer, 2015;81: 325-336. Doi: https://doi.org/10.1016/j.ijheatmasstransfer.2014.10.018 